PROCEEDINGS OF THE

AMERICAN MATHEMATICAL SOCIETY

Volume 100, Number 2, June 1987

\title{
WU CLASSES
}

\author{
ROBERT STONG AND TOSHIO YOSHIDA
}

\begin{abstract}
This paper shows that a nonbounding closed manifold with Wu classes $v_{i}=0$ for $i>k$ has dimension bounded by some function of $k$.
\end{abstract}

1. Introduction. Let $M^{n}$ be a closed $n$-dimensional manifold. By Poincaré duality, there are unique classes $v_{i} \in H^{i}\left(M ; Z_{2}\right)$ satisfying $v_{i} x[M]=\mathrm{Sq}^{i} x[M]$ for all $x \in H^{n-i}\left(M ; Z_{2}\right)$. Since $\mathrm{Sq}^{i} x=0$ for $i>n-i, v_{i}=0$ for $i>[n / 2]$, and the class

$$
v=1+v_{1}+v_{2}+\cdots+v_{[n / 2]} \in H^{*}\left(M ; Z_{2}\right)
$$

is called the total Wu class of $M$.

The Stiefel-Whitney class $w=1+w_{1}+w_{2}+\cdots+w_{n}$ of $M$ is given by $w=\operatorname{Sq} v$, where $\mathrm{Sq}=1+\mathrm{Sq}^{1}+\mathrm{Sq}^{2}+\cdots$, or equivalently $w_{i}=\sum_{j=0}^{i} \mathrm{Sq}^{i-j} v_{j}$. It is well known that the Stiefel-Whitney numbers $w_{i_{1}} \cdots w_{i_{r}}[M]$ determine the cobordism class of $M$. In particular, if $n>0$ and $M$ is not a boundary, there must be an $i>0$ for which $v_{i} \neq 0$.

The purpose of this note is to show that $v_{i} \neq 0$ for some moderately large value of $i$, and specifically to prove the following proposition.

Proposition. For any integer $k$ there is an integer $N=N(k)$ so that a closed manifold $M$ with Wu class $v=1+v_{1}+\cdots+v_{k}$ bounds if $\operatorname{dim} M>N$.

No analogous result holds if one considers other characteristic classes. In particular, one has the following.

OBSERVATION. There is a nonbounding closed manifold of dimension $2^{r+1}$ with Stiefel-Whitney class $w=1+w_{1}+w_{2}$.

OBSERVATION. There is a nonbounding closed manifold of dimension $2^{s}-2$ with normal Stiefel-Whitney class $\bar{w}=1 / w=1+\bar{w}_{1}$.

The authors are indebted to the Japanese Government and to the National Science Foundation for financial support during this work.

2. The proof. In order to prove the proposition, one makes use of the space $B O$ which classifies stable vector bundles. One then has $H^{*}\left(B O ; Z_{2}\right)=Z_{2}\left[w_{i} \mid i \geq 1\right]$, where $w_{i}$ is the universal Stiefel-Whitney class. One has

LEMMA. Let $B_{k}^{*}=H^{*}\left(B O ; Z_{2}\right) / I\left(v_{i} \mid i>k\right)$, where $I\left(v_{i} \mid i>k\right)$ is the ideal generated over the Steenrod algebra by the classes $v_{i}, i>k$, where $v=\operatorname{Sq}^{-1} w$. Then $B_{k}^{*}$ is finite dimensional.

The proposition is then immediate by taking $N(k)$ to be the largest integer $i$ for which $B_{k}^{i} \neq 0$.

Received by the editors March 6, 1986.

1980 Mathematics Subject Classification (1985 Revision). Primary 57R20. 
To prove the lemma, one may proceed by induction on $k$. For $k=1, B_{1}^{*}$ has $v=1+v_{1}$ and $w=\operatorname{Sq} v=1+v_{1}+v_{1}^{2}$ with

$$
0=\operatorname{Sq}^{1}\left(v_{1}^{2}\right)=\operatorname{Sq}^{1} w_{2}=w_{3}+w_{2} w_{1}=w_{2} w_{1}=v_{1}^{3} \text {. }
$$

Thus $B_{1}^{*}=Z_{2} \oplus Z_{2} \oplus Z_{2}$ with basis $1, v_{1}, v_{1}^{2}$. One then assumes $B_{j}^{*}$ is finite dimensional for all $j<k$, and considers $B_{k}^{*}$ in which $v=1+v_{1}+\cdots+v_{k}$ and

$$
w=1+v_{1}+\left(v_{2}+v_{1}^{2}\right)+\cdots+\left(\mathrm{Sq}^{k-1} v_{k}\right)+\left(v_{k}^{2}\right)
$$

Step 1. There is an integer $r$ for which $v_{k}^{2^{r}}=0$. For $k$ odd, one has

$$
\mathrm{Sq}^{1} w_{2 k-1}=w_{1} w_{2 k-1}
$$

but

$$
\mathrm{Sq}^{1} w_{2 k-1}=\mathrm{Sq}^{1} \mathrm{Sq}^{k-1} v_{k}=\mathrm{Sq}^{k} v_{k}=v_{k}^{2}=w_{2 k}
$$

Also

$$
\mathrm{Sq}^{1} w_{2 k}=\operatorname{Sq}^{1}\left(v_{k}^{2}\right)=0
$$

but

$$
\mathrm{Sq}^{1} w_{2 k}=w_{2 k+1}+w_{1} w_{2 k}=w_{1} w_{2 k}
$$

Thus

$$
v_{k}^{4}=w_{2 k} w_{2 k}=w_{2 k} w_{1} w_{2 k-1}=0 \cdot w_{2 k-1}=0 .
$$

Now suppose $k$ is even. One then has

$$
0=\mathrm{Sq}^{2 j+1}\left(v_{k}^{2}\right)=\mathrm{Sq}^{2 j+1} w_{2 k}=w_{2 j+1} w_{2 k}=w_{2 j+1} v_{k}^{2} .
$$

Let $I_{r}=\left\{\alpha \in B_{k}^{*} \mid \alpha v_{k}^{2^{r}}=0\right\}$. One then has $I_{0} \subset I_{1} \subset I_{2} \subset \cdots$ and lets $I=$ $\bigcup_{r=0}^{\infty} I_{r}$.

Claim. $I$ is an ideal closed under the action of the Steenrod algebra.

ProOF. If $\alpha \in I, \beta \in B_{k}^{*}$, there is an $r$ with $\alpha \in I_{r}$ so $\alpha v_{k}^{2^{r}}=0$. Then $\alpha \beta v_{k}^{2^{r}}=0$ so $\alpha \beta \in I_{r}$ giving $\alpha \beta \in I$. If $\alpha \in I$ and $j$ is an integer, one wants to see that $\mathrm{Sq}^{j} \alpha \in I$. Since $\alpha \in I$, there is an $r$ with $\alpha \in I_{r}$ or $\alpha v_{k}^{2^{r}}=0$. Letting $s>r$ with $2^{s}>j$ one has

$$
\mathrm{Sq}^{j} \alpha \cdot v_{k}^{2^{s}}=\mathrm{Sq}^{j}\left(\alpha v_{k}^{2^{s}}\right)=\mathrm{Sq}^{j}\left(\alpha v_{k}^{2^{r}} \cdot v_{k}^{2^{s}-2^{r}}\right)=\mathrm{Sq}^{j}(0)=0,
$$

so $\mathrm{Sq}^{j} \alpha \in I_{s}$ and $\mathrm{Sq}^{j} \alpha \in I$.

One now considers $B_{k}^{*} / I$ in which $w_{2 q+1}=0$ since, $w_{2 q+1} \in I_{1} \subset I$. As is well known $[\mathrm{M}], H^{*}\left(B O ; Z_{2}\right)$ modulo the ideal generated by the odd $w_{i}$ is isomorphic to $H^{*}\left(B U ; Z_{2}\right)$, which is isomorphic to $H^{*}\left(B O ; Z_{2}\right)$, but with all dimensions formally doubled. Thus, one has a homomorphism

$$
B_{k / 2}^{*} \rightarrow B_{k}^{*} / I: w_{i} \rightarrow w_{2 i}
$$

which is epic. Inductively, $B_{k / 2}^{*}$ is finite dimensional, so $B_{k}^{*} / I$ is finite dimensional.

Thus there is an $s$ with $v_{k}^{2^{s}} \in I$, and so $v_{k}^{2^{s}} \in I_{r}$ for some $r$, or $v_{k}^{2^{s}} \cdot v_{k}^{2^{r}}=0$. There is then a $q$ with $v_{k}^{2^{q}}=0$, completing the proof of Step 1 .

Step 2. There is an integer $q$ so that $x^{2^{q}}=0$ for all $x \in B_{k}^{*}, x \neq 1$.

To see this, one chooses $r$ from Step 1 so that $v_{k}^{2^{r}}=0$ and considers

$$
\phi: B_{k}^{*} \rightarrow B_{k}^{*}: x \rightarrow x^{2^{r}}
$$


The kernel of this homomorphism is an ideal over the Steenrod algebra. If $\phi(x)=0$, then $\phi(x y)=\phi(x) \phi(y)=0$ and $\phi\left(\operatorname{Sq}^{i} x\right)=\left(\operatorname{Sq}^{i} x\right)^{2^{r}}=\operatorname{Sq}^{2^{r} i}\left(x^{2^{r}}\right)=\operatorname{Sq}^{2^{r} i}(0)=$ 0 . Since $\phi\left(v_{k}\right)=0, \phi$ factors through $B_{k-1}^{*}$, and by induction $B_{k-1}^{*}$ is finite dimensional, so $\phi\left(B_{k}^{*}\right)$ is finite dimensional. Thus, there is an $m$ so that $\phi\left(B_{k}^{*}\right)^{i}=0$ if $i>m$ and in particular, there is an $s$ with $2^{s}>m$, so that for any $x \in B_{k}^{*}$, $x^{2^{r+s}}=\phi\left(x^{2^{s}}\right)=0$ being in image $\phi$ and having dimension larger than $m$. Taking $q=r+s$ gives the desired assertion.

Finally, $B_{k}^{*}$ is generated by $w_{1}, w_{2}, \ldots, w_{2 k}$ and has $w_{i}^{2^{q}}=0$ for each $i$, so $B_{k}^{j}=0$ if $j \geq 2^{q}(1+2+\cdots+2 k)=2^{q}\left(\begin{array}{c}2 k+1 \\ 2\end{array}\right)$. Thus $B_{k}^{*}$ is finite dimensional.

3. Comments. Comment 1 . The manifold $R P^{2^{s}-2}$ is nonbounding and has $\bar{w}=1+\bar{w}_{1}$, so no analogous result holds for the normal Stiefel-Whitney class.

Comment 2. The manifold $M^{2^{r+1}}=R P\left(\xi_{1} \oplus \xi_{2} \oplus 1\right)$ fibered over $R P^{2^{r}-1} \times$ $R P^{2^{r}-1}$ with fiber $R P^{2}, \xi$ being the nontrivial line bundle over the $i$ th factor, is nonbounding and has $w=1+w_{1}+w_{2}$. Hence, no analogous statement holds for the Stiefel-Whitney class.

Comment 3. It would be of interest to find the smallest value of $N(k)$ which has the property described in the proposition. One has a prospect of tracing through the argument to find a bound, but that will be too large. In fact, $N(k)$ can be less than the largest $i$ for which $B_{k}^{i} \neq 0$.

Unpleasnt calculations give $N(1)=2, N(2)=5, N(3)=7$, which coincides with $3 k-\alpha(k)$, where $\alpha$ is the number of one's in the 2 -adic expansion. That bound does not work generally (seemingly $N(6) \geq 19$ ).

Comment 4. One potential application is given by

Proposition. If $N(k) \leq 4 k$ for $k \leq[(j-1) / 2]$, then every closed $M^{n}$ which fibers over the sphere $S^{j}$ with $n<2 j$ is a boundary.

PROOF. If $F^{n-j} \stackrel{i}{\rightarrow} M^{n} \stackrel{\pi}{\rightarrow} S^{j}$ is a fibering with $n<2 j$, then $v_{i}(M)=0$ for $i>[(n-j) / 2]$.

Note. In [D], it is shown that if $M^{n}$ fibers over $S^{j}$ and $n<(3 j-2) / 2$, then $M$ bounds (see Proposition 2.4).

\section{REFERENCES}

[D] A. Didierjean, Classes de cohomologie invariantes par cobordisme et classes de cobordisme se fibrant sur des sphères, C. R. Acad. Sci. Paris 289 (1979), 165-166.

[M] J. W. Milnor, On the Stiefel-Whitney numbers of complex manifolds and of spin manifolds, Topology 3 (1965), 223-230.

Department of MAThematics, University of Virginia, ChaRlottesville, VirGINIA 22903

FACUlTy of INTEgRATEd ARTS AND SCIENCES, HiRoshima UNIVERSity, HiRoshima 730, JAPAN 